\title{
Genes for defense response to Plasmodiophora brassicae during late infection in small spheroid galls of Brassica rapa
}

\author{
H. YANG ${ }^{1,2}$, X.Y. FANG ${ }^{2}$, X.L. WANG ${ }^{2}$, J. ZHENG ${ }^{2}$, C.L. YI ${ }^{2}$, J. FAN ${ }^{1}$, S. YUAN ${ }^{3}$, J. SHANG ${ }^{2}$, \\ I.Y. HUANG ${ }^{2}$, and W.M. WANG ${ }^{1 *}$ \\ Rice Research Institute and Research Center for Major Crop Diseases, Sichuan Agricultural University, \\ Chengdu 611130, P.R. China ${ }^{1}$ \\ College of Agronomy, Sichuan Agricultural University, Chengdu 611130, P.R. China ${ }^{2}$ \\ College of Resources, Sichuan Agricultural University, Chengdu 611130, P.R. China ${ }^{3}$
}

\begin{abstract}
Plasmodiophora brassicae is a biotrophic pathogen causing clubroots of cruciferous crops. The Brassica rapa accession T1-145 has an ability to produce small spheroid galls (SSGs), which represent neither a fully compatible interaction nor a complete resistance. To explore the defense response in SSGs induced by P. brassicae infection, global transcriptome profiling SSGs was performed at different time points. By comparing gene expression patterns, we identified many defense related genes. The first group included genes encoding receptor-like protein/kinases, such as cysteine-rich receptor-like protein kinases, receptor-like proteins, phloem intercalated with xylem/tracheary element differentiation inhibitory factor receptor $(P X Y / T D R), P X Y$-correlated 1, wall-associated kinases, nuclear shuttle protein-interacting kinase, lectin receptorlike kinases, and flagelin-sensitive 2, which might activate a basal defense. The second group involved robust effectortriggered immunity response genes such as resistance to leptosphaeria maculans $1 B$, constitutive shade-avoidance 1 , target of avirulence B operation 1, ribosomal protein of the small subunit 6, resistance to Pseudomonas maculicola 1 -interacting protein 4 , enhanced disease resistance $2 L$, and recognition of Peronospora parasitica 13-like protein 4 . The third group included genes encoding secondary cell wall formation related protein/s, a nodulin-like protein, a germin-like protein, a jacalin-related lectin, a defensin-like protein, tumor inhibitors, and sugars will eventually be exported transporter, which might contribute to quantitative resistance against $P$. brassicae. The gene expressions were the highest at the late stage of infection. To our knowledge, it is the first report on exploring defense response genes during SSG occurrence by a transcriptome analysis. Our data would provide useful information to further explore molecular mechanisms of the incomplete resistance.
\end{abstract}

Additional key words: biotrophic pathogen, effector-triggered immunity, gene expression patterns, receptor-like protein/kinases.

\section{Introduction}

The clubroot disease in cruciferous crops is caused by Plasmodiophora brassicae, a soil borne and obligate biotrophic pathogen (Crisp et al. 1989). The disease is spreading rapidly in Asia and has become an emerging problem in many countries. Clubroot disease is difficult to be controlled by either chemicals or cultural practices. The development of a resistant cultivar is believed to be the most effective and environmentally friendly option. Previous reports pointed out that clubroot resistance $(\mathrm{CR})$ is controlled by a single resistance gene in B. rapa (Piao et al. 2009). Quantitative trait loci involved in B. rapa $\mathrm{CR}$ were also studied (Suwabe et al. 2006). The effectiveness of $C R$

Submitted 29 July 2019, last revision 9 January 2020, accepted 10 February 2020.

Abbreviations: AvrB - avirulence B; CDPK - calcium-dependent protein kinase; CR - clubroot resistance; CRK - cysteine-rich receptorlike protein kinase; dpi - days post inoculation; ET - ethylene; GO - gene ontology; JA - jasmonic acid; KEGG - Kyoto encyclopedia of genes and genomes; LecRK - lectin receptor-like kinase; LRK - lectin receptor kinase; MYB - myeloblastosis; NIK - nuclear shuttle protein-interacting kinase; PR - pathogenesis-related protein; PXC - phloem intercalated with xylem-correlated; RIN - RPM1interacting protein; RLK - receptor-like protein kinase; RLP - receptor-like protein; RNA-seq - sequence of RNA; RPM - resistance to Pseudomonas syringae pv. maculicola; RPS - ribosomal protein of the small subunit; SA - salicylic acid; SF - Shifang; SRF - strubbelig receptor family; SSG - small spheroid gall; SWEET - sugars will eventually be exported transporter; TAO - target of AvrB operation; TCP - teosinte branched1/cycloidea/pcf; TDR - tracheary element differentiation inhibitory factor receptor; WAK - wall-associated kinase; WOX - wuschel homeobox related; XC - Xichang.

Acknowledgement: The study was financed by the Innovation Experiment of Sichuan Province (Project number 201910626064).

The first three authors contributed equally to this work.

* Corresponding author; e-mail: j316wenmingwang@sicau.edu.cn 
genes has been tested against some different pathotypes. For example, $\mathrm{CRa}, \mathrm{CRb}, \mathrm{CRk}, \mathrm{Crr} 1, \mathrm{Crr} 3$, and Crr4 were resistant to pathotype 2 in B. rapa (Suwabe et al. 2006). $P$. brassicae populations in the fields are often a mixture of different pathotypes (Xue et al. 2008). P. brassicae is a highly variable pathogen and its genotyping revealed the presence of distinct populations (Holtz et al. 2018). Due to the continuous cropping of a single resistant cultivar, the virulent pathotypes are likely to be selected and then compromise the resistance (Tanaka et al. 2013). Therefore, it is necessary to generate a cultivar resistant to the broad spectrum of $P$. brassicae races.

The life cycle of $P$. brassicae has three phases: survival in the soil, root hair infection, and cortical infection phase (Kageyama and Asano 2009). The cortical infection stage is crucial to gall development in roots, which creates a strong metabolite sink to attract assimilates from other tissues (Ludwig-Müller 2014). Small spheroid galls (SSGs) are different in size and form from the typical spindle galls of clubroots. The SSGs are generally regarded as a resistance form, however, the resting spores can be also observed in SSGs; they represent neither complete resistance, nor full susceptibility (Osaki et al. 2008, Rennie et al. 2013, Peng et al. 2016).

A previous report demonstrated the difference between symptomless roots and gall tissue in Brassica oleracea by transcriptome analysis (Ciaghi et al. 2019). Jubault et al. (2013) also pointed out that the partial resistance to clubroot in Arabidopsis is due to a reduction or delay in the host primary metabolism and induction of an earlier or stronger plant defense response. However, the transcriptome analyses were mainly focused on the early infection phase (Jubault et al. 2013, Chen et al. 2016, Zhao et al. 2017). In this study, we focused on the different infection periods, including root hair infection phase (primary stage), cortical infection phase (secondary stage), and the symptom development phase, to discover which stage was the key one and which defense pathways might be involved in the restricted development of galls in $B$. rapa genotype T1-145, using a transcriptome analysis. We also wanted to explore the main regulatory pathways on which the specific incomplete resistance in SSG depends. This would provide more references for revealing the resistance mechanism of the host against $P$. brassicae.

\section{Materials and methods}

Preparation of resting spore suspension: For preparation of plant inoculations, Plasmodiophora brassicae resting spores were prepared as previously described (Ji et al. 2014). Root galls were homogenized using $10 \%(\mathrm{~m} / \mathrm{v})$ sucrose in a blender. The slurry was filtered through 8 layers of gauze and the suspension was clarified by centrifugation at $3000 \mathrm{~g}$ for $9 \mathrm{~min}$. The pellet was suspended in sterile water and transferred into a new tube containing $40 \mathrm{~cm}^{3}$ of water. After centrifugation at $3000 \mathrm{~g}$ for $12 \mathrm{~min}$, the resting spores precipitate was resuspended in sterile water, and adjusted to a concentration of $1 \times 10^{7}$ (spores) $\mathrm{cm}^{-3}$, and stored at $4{ }^{\circ} \mathrm{C}$. For pathotype determination according to the Williams, 30 seedlings from differential hosts were inoculated and three replicates were taken for the pathotype determination. The Williams identification hosts Jersey Queen and Badger shipper cultivars of Brassica oleracea var. capitata, and Laurentian and Wilhelmsburger cultivars of Brassica napobrassica were inoculated with $P$. brassicae resting spores. The judgment standard of pathotype was set accorded to previous report (Williams 1996).

Plasmodiophora brassicae inoculation: Seeds of Chinese cabbage (Brassica rapa L.) genotype T1-145 were sterilized with $75 \%(\mathrm{v} / \mathrm{v})$ ethanol for $5 \mathrm{~min}$ and then repeatedly washed with sterile water. After seedlings grew for one week, or cotyledons were fully expanded, $0.5 \mathrm{~cm}^{3}$ of spore suspension of the Xichang (XC) isolate (from Xichang in Sichuan Province, China) or the Shifang (SF) isolate (from Shifang in Sichuan Province, China) was inoculated at the base of each seedling. Resting spores were diluted to a density of $10^{7}$ spores $\mathrm{cm}^{-3}$ in sterile distilled water. The $\mathrm{XC}$ isolate and SF isolate were both pathotype 4 based on Walliams identification system. The inoculated plants were maintained in a growth chamber under a 16-h photoperiod, an irradiance of $120 \mu \mathrm{mol} \mathrm{m} \mathrm{m}^{-2} \mathrm{~s}^{-1}$, and a temperature of $25^{\circ} \mathrm{C}$, and the soil was kept moist during the treatment period. The roots were harvested at $4,10,16$, and $45 \mathrm{~d}$ post inoculation (dpi) and were washed thoroughly with distilled water to remove the spores adsorbed onto the surface. They were then frozen in liquid nitrogen and stored at $-70{ }^{\circ} \mathrm{C}$. Total RNA of the clubroot was extracted and sent for sequencing.

Analysis of RNA sequence (RNA-seq): The RNA was extracted from two biologically different root samples using TRIzol®) reagent (Life Technologies, Carlsbad, CA, USA) following the manufacturer's protocol. The RNA quantity and quality were determined using a 2100 BioAnalyzer (Agilent Technologies, Santa Clara, USA). The RNA libraries were constructed with $2 \mu \mathrm{g}$ of total RNA and subjected to high-through-put sequencing by a HiSeq-4000 sequencer (Illumina, San Diego, CA, USA). Approximately $10 \mathrm{Gbp}$ of reads for each sample were obtained. The filter software $N g q c$ was used to analyze the quality of the raw reads, after filtering the raw reads by removing adapter and reads with unknown base ' $\mathrm{N}$ ' content greater than $5 \%$.. Sequencing reads were compared to the reference database for the B. rapa genome (http:// brassicadb.org/brad/) using Cufflinks software and the length statistics of reference transcriptome sequences were performed with perl scripts (Trapnell et al. 2009). Nonemapping sequences were regarded as novel genes.

The fragments per kilobase million method was used to calculate the normalized expression data of each gene (Mortazavi et al. 2008). The $R$ language software was used for the generation of heatmap (Audic and Claverie et al. 1997).

Differentially expressed genes (DEGs) were selected by the DEseq 2 software, with the criteria of absolute Log2 (fold change) $\geq 2$ and false discovery rate $\leq 0.01$ between any two samples (Wang et al. 2010). The fragments per 
kilobase million of genes were log-converted. The value of Log2 (fragments per kilobase million +1) for each transcript was received.

The Gene ontology $(G O)$ database was used for gene ontology analysis (https://en.wikipedia.org/ wiki/ Hypergeometric_distribution) and $G O$ enrichment analysis was performed using GOseq (Young et al. 2010). Unigene sequences were compared with the nonredundant protein database and $G O$ (Ashburner et al. 2000). To elucidate the metabolic pathways of these predicted genes, the Kyoto encyclopedia of genes and genomes $(K E G G)$ pathways of DEGs were compared (Wu et al. 2006).

Reverse transcription quantitative PCR analysis: Root samples were collected at 4, 10, 16, and $45 \mathrm{dpi}$. The cDNA was synthesized from $0.5 \mu \mathrm{g}$ of total RNA using ReverTraAce qPCR RT Master Mix with gDNA remover (Toyobo, Osaka, Japan). A two-step real-time PCR reaction was performed using a QuantiFast ${ }^{\mathrm{TM}} S Y B R^{\circledR}$ qPCR kit (Qiagen, Hilden, Germany) with $100 \mathrm{ng}$ template DNA and $10 \mathrm{nM}$ of each primer in a final volume of $0.01 \mathrm{~cm}^{3}$, according to the following protocol: polymerase activation at $95{ }^{\circ} \mathrm{C}$ for $5 \mathrm{~min}$ followed by denaturation at $95{ }^{\circ} \mathrm{C}$ for $10 \mathrm{~s}$, and concurrent annealing and extension at $65^{\circ} \mathrm{C}$ for $30 \mathrm{~s}$. The $B$. rapa actin ( $A C T 2)$ gene was used as an internal control. Primer sequences are listed in Table 1 Suppl.

\section{Results and discussion}

Comparative analysis of $P$. brassicae infection in $B$. rapa: The T1-145 genotype was susceptible to XC isolate and developed the typical spindle galls. A special interaction (T1-145 inoculated with SF isolate) consistently resulted in SSG production (Fig. 1). The plasmodia in the root hair and cortical tissue had no difference between SF and XC infections at 4 and 10 dpi. Many sporangia were obviously empty in the sample of SF infection at $16 \mathrm{dpi}$, but the resting spores could be observed in the SSGs and spheroid galls at $45 \mathrm{dpi}$. However, by observation of the ultrastructure of bare-handed section, we could observe that the resting spores of $\mathrm{XC}$ isolates were more concentrated and so the number of $\mathrm{XC}$ spores in a cell was higher than in the case of SF isolate (Fig. 1 Suppl.).

The RNA-seq analysis: The samples of T1-145 after SF isolate and $\mathrm{XC}$ isolate infection at $4,10,16$, and $45 \mathrm{dpi}$ were compared by a transcriptome analysis. Clean reads were in the range from 62860626 to 85058774 , and clean bases ranged from 9 to $12 \mathrm{Gbp}$. Data from the two biological replicates were highly consistent according to principal components analysis, which indicated that no obvious bias in the cDNA library construction between the two biological replicates. The guanine and cytosine (GC) values of sequence data from the 24 libraries ranged from 45 to $50 \%$, the quality of clean reads Q30\% were all $>89 \%$, and the quality of clean reads Q20\% were all $>95 \%$. Therefore, the quality of the sequencing data was sufficient for further analysis. An overview of the sequencing data is shown in Table 2 Suppl.
Functional annotation of DEGs: A total of $809 \mathrm{GO}$ terms in $B$. rapa by comparing SF isolate with $\mathrm{XC}$ isolate were assigned to three classes (corrected $P$ value $<0.05$ ): 'biological process', 'cellular component', and 'molecular function'. The top ten significantly enriched $G O$ terms at each time point were listed on Table 3 Suppl. For instance, in the 'molecular function' class, $G O$ terms 'cofactor binding' and 'aldehyde-lyase activity' at $4 \mathrm{dpi}$, 'NAD binding' at 10 dpi, 'microtubule motor activity', 'microtubule binding', 'tubulin binding', 'motor activity' and 'cytoskeletal protein binding' at 45 dpi were specially enriched.

To identify the biological pathways involved in defense against $P$. brassicae in $B$. rapa, we analyzed the differentially expressed unigenes in $K E G G$, the top 25 enriched $K E G G$ pathways are listed in Table 1 (corrected $P$ value $<0.05)$. 'Ribosome' $(813,22.66 \%$ ) was the most common term, followed by 'starch and sucrose metabolism' (167, 4.65\%), 'amino sugar and nucleotide sugar metabolism' (158, 4.40\%). Previous reports indicated that $P$. brassicae infection caused significant increase of glucose and fructose content, also suggesting that sugar translocation and $P$. brassicae growth are closely related (Li et al. 2018). The KEGG of 'plant hormone signal transduction' $(285,7.95 \%)$ was also obviously enriched. The clubroot disease pointed to an involvement of plant hormones in clubroot symptom development, such as cytokinins and auxin. Cytokinin oxidase/dehydrogenase overexpressing plants were disease resistant (Devos et al. 2006, Siemens et al. 2006). These pathways might be partly contribute to the SSG development.

The number of responsive genes is different between $\mathrm{SF}$ and $\mathrm{XC}$ isolate inoculations in $B$. rapa T1-145: To identify the genes showing the most differential response to $\mathrm{SF}$ and $\mathrm{XC}$ isolate infections, we selected the differentially expressed genes (DEGs) at variations of absolute $\log 2 \geq 2$ and false discovery rate $\leq 0.01$ at every time point. Totally 6359 DEGs were identified. Comparing the sample of SF infection with the control and the sample of XC infection with the control, there were more up-regulated genes at 4, 16, and 45 dpi than at 10 dpi (Fig. 2A,B). Next we compared the number of DEGs between the samples of $\mathrm{SF}$ and $\mathrm{XC}$ isolate infections. The numbers of up-regulated genes were 469, 162, 44, and 1706 at 4, 10, 16, and $45 \mathrm{dpi}$,
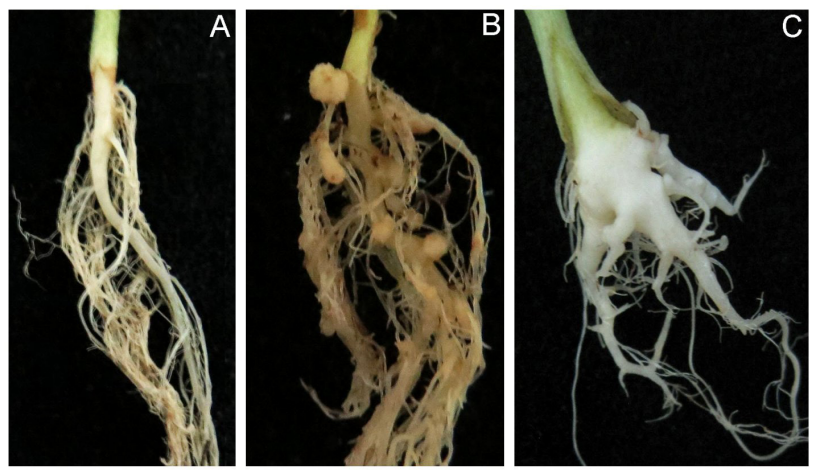

Fig. 1. Morphology differences of Chinese cabbage plants. The healthy root $(A)$, the root with small spheroid galls $(B)$, the root with spindle galls $(C)$. 
Table 1. Top 25 enriched Kyoto encyclopedia of genes and genomes pathways. DEGs - differentially expressed genes, SF - Shifang isolate, $\mathrm{XC}$ - Xichang isolate.

\begin{tabular}{|c|c|c|c|}
\hline Pathway & The number of DEGs & Pathway ID & $\mathrm{SF} v s . \mathrm{XC}$ \\
\hline Ribosome & $813(22.66 \%)$ & ath03010 & up \\
\hline Starch and sucrose metabolism & $167(4.65 \%)$ & ath00500 & up \\
\hline Amino sugar and nucleotide sugar metabolism & $158(4.40 \%)$ & ath00520 & up \\
\hline Plant hormone signal transduction & $285(7.95 \%)$ & ath04075 & up/down \\
\hline Plant-pathogen interaction & $141(3.93 \%)$ & ath04626 & up/down \\
\hline Oxidative phosphorylation & $110(3.07 \%)$ & ath00190 & up \\
\hline Carbon fixation in photosynthetic organisms & $89(2.48 \%)$ & ath00710 & up \\
\hline Carbon metabolism & $84(2.34 \%)$ & ath01200 & up \\
\hline Glyoxylate and dicarboxylate metabolism & $63(1.76 \%)$ & ath00630 & up/down \\
\hline Photosynthesis & $50(1.39 \%)$ & ath00195 & up \\
\hline Proteasome & $45(1.25 \%)$ & ath03050 & up \\
\hline Glycine, serine, and threonine metabolism & $42(1.17 \%)$ & ath00260 & up \\
\hline Alanine, aspartate, and glutamate metabolism & $30(0.84 \%)$ & ath00250 & up \\
\hline Fatty acid metabolism & $29(0.81 \%)$ & ath01212 & up \\
\hline Fatty acid biosynthesis & $22(0.61 \%)$ & ath00061 & up \\
\hline Biosynthesis of secondary metabolites & $231(6.44 \%)$ & ath01110 & down \\
\hline Protein processing in endoplasmic reticulum & $192(5.35 \%)$ & ath04141 & down \\
\hline Phenylpropanoid biosynthesis & $163(4.55 \%)$ & ath00940 & down \\
\hline Biosynthesis of amino acids & $161(4.49 \%)$ & ath01230 & down \\
\hline Phenylalanine metabolism & $127(3.54 \%)$ & ath00360 & down \\
\hline RNA transport & $117(3.26 \%)$ & ath03013 & down \\
\hline Endocytosis & $101(2.82 \%)$ & ath04144 & down \\
\hline Phenylalanine, tyrosine, and tryptophan biosynthesis & $40(1.12 \%)$ & ath00400 & down \\
\hline Glycerolipid metabolism & $33(0.92 \%)$ & ath00561 & down \\
\hline$\alpha$-Linolenic acid metabolism & $33(0.92 \%)$ & ath00592 & down \\
\hline
\end{tabular}

respectively. The numbers of down-regulated genes were $142,655,245$, and 791 at $4,10,16$, and 45 dpi, respectively (Fig. 2C). Obviously, there were more up-regulated genes at $45 \mathrm{dpi}$ than the other time points, which indicated that the late infection period might be critical for maintaining the defense against $P$. brassicae.

To identify which genes were most likely associated with the resistance, we classified the DEGs into tree types. The first type: the gene expressions were up-regulated in both sample of SF isolate infection [ $\log 2$ (SF vs. control) $\geq 2]$ and $\mathrm{XC}$ isolate infection [ $\log 2$ ( $\mathrm{XC} v s$. control) $\geq 2$ ] at a time point by comparing with control. Due to the up-regulation of gene expression under the infection of $P$. brassicae, we suggested that most of these genes

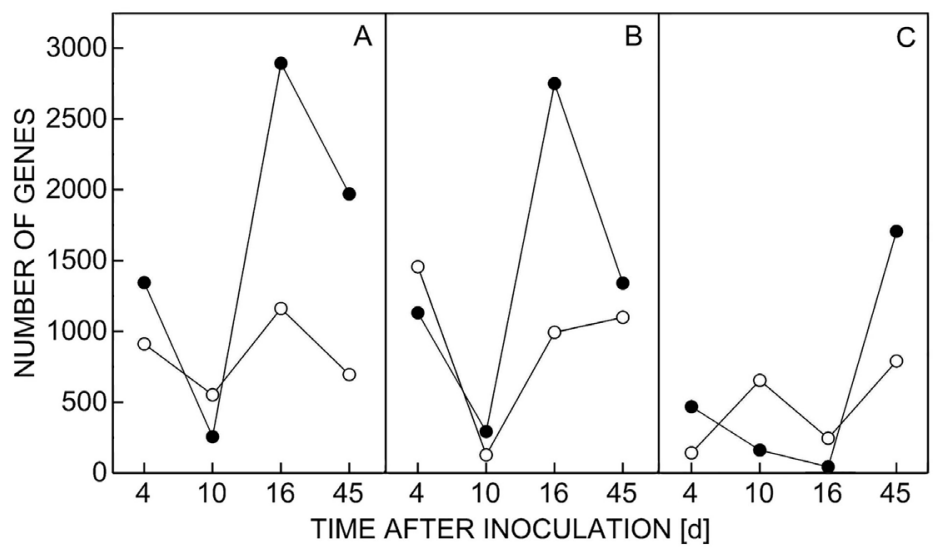

Fig. 2. The number of differentially expressed genes. Numbers of up-regulated (closed circles) and down-regulated (open circles) genes by comparing samples of Shifang (SF) isolate infection with a control at $4,10,16$, and 45 days post inoculation (dpi, $A$ ), by comparing samples of Xichang (XC) isolate infection with a control at 4, 10,16, and $45 \mathrm{dpi}(B)$, and by comparing samples of SF infection with $\mathrm{XC}$ infection at $4,10,16$, and $45 \mathrm{dpi}(C)$. 
might be associated with the basic resistance of host against $P$. brassicae or contributing to sustained growth of $P$. brassicae.

The second type: the gene expressions were lower in the sample of SF isolate infection than $\mathrm{XC}$ isolate infection. 1) 569 DEGs were selected at variations of absolute Log2 $(\mathrm{SF} v s . \mathrm{XC}) \leq-2$ at a time point and $-1 \leq \mathrm{Log} 2$ (SF vs. XC) $\leq 1$ at the other time points. 2) 440 DEGs were selected at variations of absolute $\log 2(\mathrm{SF} v s$. XC) $\leq-2$ at a time point and $\log 2(\mathrm{SF} v s . \mathrm{XC}) \leq-1$ at the other time points. Due to the high-expression of DEGs with $\mathrm{XC}$ isolate infection, the second type of DEGs might be beneficial to the development of the typical spindle galls of clubroots.

The third type: The gene expressions were obviously higher in the sample of SF isolate infection than XC isolate infection and 2232 DEGs were finally obtained $(\log 2(\mathrm{SF} v s . \mathrm{XC}) \geq 2)$ at a time point. The third type of DEGs might be important for the host defense against the P. brassicae $\mathrm{SF}$ isolate.

Expression patterns over the time course of infection classified genes contributing to the defense: We suggest that the third type genes might be associated with the host defense. To be more accurate in demonstrating the post-invasive defense, we further performed analysis on the expression patterns of 2232 DEGs. We classified the third type of DEGs into four groups: Group A including 355 genes fits the absolute $\log 2$ (SF vs. XC) $\geq 2$ only during the root hair infection period (at $4 \mathrm{dpi}$ ). Group B including 151 genes fits the absolute $\log 2$ (SF vs. XC) $\geq 2$ only during the cortical infection period (at 10 and 16 dpi). Group C including 1580 genes fits the absolute Log2 (SF vs. XC) $\geq 2$ only during the symptomatic period (at 45 dpi). Group D including 146 genes fits the absolute Log2 (SF vs. XC) $\geq 2$ during root hair, cortical infection or symptomatic period.

To confirm the evaluation of RNA-seq data, we repeated the time course experiment and collected samples for the reverse transcription quantitative PCR on six representative genes (Fig. 3).

Differentially expressed genes involved in the resistance to $P$. brassicae: The third type DEGs were used to analyze the defense related genes. All detailed data are listed on the website https://share.weiyun.com/U3b0imIV, password: 6utmqg, and some representative data are shown in Fig. 4. The resistance proteins are crucial to detect pathogen effectors and trigger the subsequent defense responses. Previous work has shown that the Arabidopsis target of avirulence $\mathrm{B}$ operation 1 (TAO1) is a toll/interleukin 1 receptor-nucleotide binding leucine-rich repeat receptorlike kinase protein, which contributes to disease resistance induced by the Pseudomonas syringae effector AvrB (Eitas et al. 2008). Ribosomal protein of the small subunit 6 (RPS6) was found conferring resistance to wheat stripe rust in barley (Li et al. 2016). Arabidopsis resistance to Pseudomonas syringae pv. maculicola-interacting protein 4 (RIN4) regulates plant immune responses to pathogenassociated molecular patterns and type III effectors (Sun et al. 2014). The disease resistance genes resistance to leptosphaeria maculans $1 B$, constitutive shade-avoidance 1, RPS6, RIN4, recognition of Peronospora parasitica 13, nonrace-specific disease resistance 1, and TAO1 were all induced, which might be involved in the defense response against $P$. brassicae.

Receptor-like protein/kinase: Cysteine-rich receptor-like protein kinases (CRKs) are one large subfamily of receptorlike protein kinases (RLKs) in Arabidopsis, which play an important role in response to abiotic and biotic stresses. Receptor-like proteins (RLPs) assume an important role for pathogen-associated molecular pattern-triggered immunity (Pitino et al.2015). Here, seven CRKs and eight RLPS were induced after SF and XC isolate infections. For example, CRK10 was induced at 4 and $45 \mathrm{dpi}$, the expression in the SF infected sample was 3-fold higher than that of

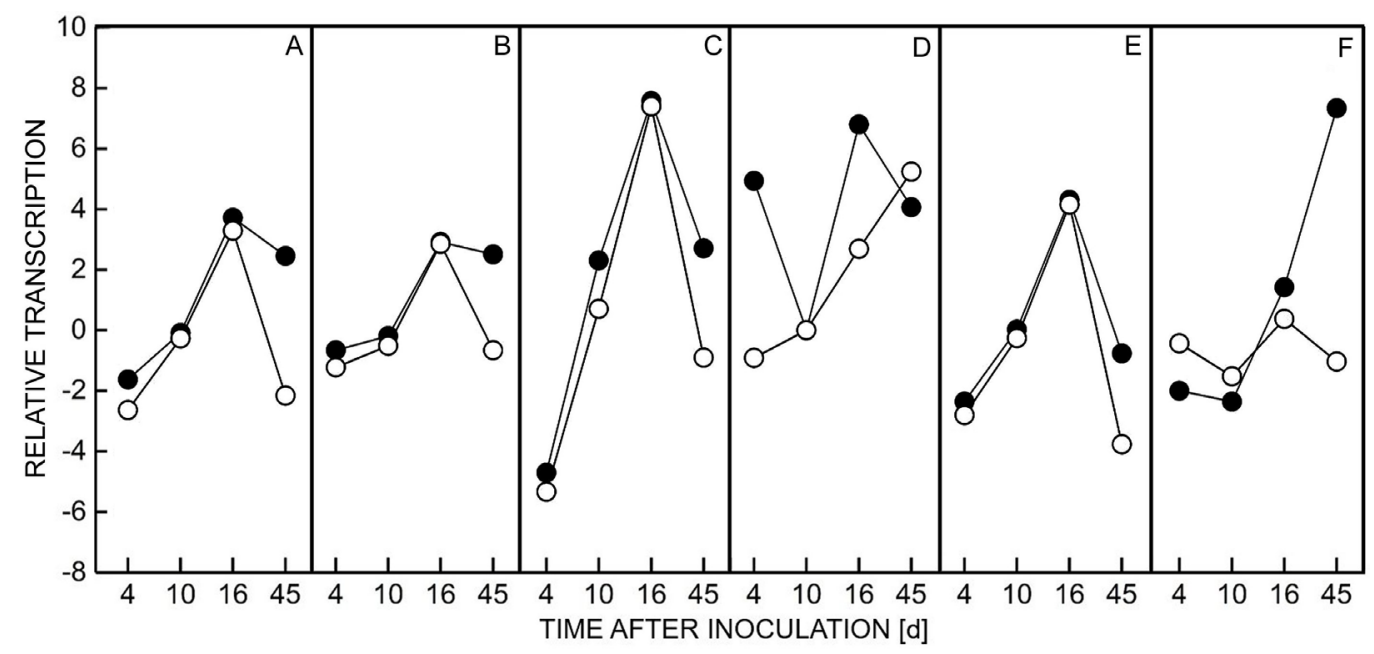

Fig. 3. Validation of data of RNA sequence by reverse transcription quantitative PCR. Relative transcriptions of phloem intercalated with xylem-correlated $1(A)$, tractheary element differentiation inhibitory factor receptor $(B)$, sugars will eventually be exported transporter $(C)$, chitinase $(D)$, defensin-like proteins $(E)$, and defensin-like proteins $(F)$ genes. Comparison of the Shifang isolate (closed circles) or the Xichang isolate (open circles) infection with a control. 
$\mathrm{XC}$ infected one at $45 \mathrm{dpi}$. The $C R K 11,12,18,40$, and 41, RLP12 (Novel00805, BraA08001180, BraA09002935, Novel00953, BraA01003449, BraA04000761), and RLP2 (BraA08003352) were induced at $16 \mathrm{dpi}$ and maintained high at 45 dpi after SF infection.

Lectin receptor-like kinases (LecRKs) belong to a specific pattern recognition receptors family and are important players in plant innate immunity (Luo et al. 2017). Here, LRKS7, LRKS6, LRK16, and LecRK44 were up-regulated at $16 \mathrm{dpi}$, and their expressions were 2-fold higher upon SF infection than upon XC infection at $45 \mathrm{dpi}$.

Secondary vascular development requires contributions from a number of leucine-rich repeat receptor like kinases. Arabidopsis leucine-rich repeat receptor like kinase genes (phloem intercalated with xylem-correlated $(P X C) 1,2$, and 3) strongly correlate with several key regulators of vascular development, phloem intercalated with xylem/ tracheary element differentiation inhibitory factor receptor (PXY/TDR), which are involved in the secondary cell wall formation in xylem fibers (Wang et al. 2013). Here, the leucine-rich repeat receptor-like tyrosine-protein kinases PXC1 (BraA03002010), PXC2 (BraA02000006), PXC3 (BraA05004219 and BraA04002945), and TDR (BraA03004735) were up-regulated at $16 \mathrm{dpi}$ and the expression was still up-regulated at 45 dpi upon SF isolate infection. The wuschel homeobox related (WOX) 4, 11, and 13 expressions were higher after SF infection than after XC infection at 45 dpi. Therefore, the PXC1 - phloem intercalated with xylem / TDR - WOX4 signaling pathway probably regulates the maturation of interfascicular fiber cells and the initiation of secondary cell wall deposition to affect $P$. brassicae extension.

Wall-associated kinases (WAKs) have been shown to be positive regulators of fungal disease resistance in several plant species (Delteil et al. 2016). Here, WAK5 (BraA08003146 and BraA06001040) and WAK-like 16 (BraA02002703) were up-regulated at $16 \mathrm{dpi}$; their expressions were higher after SF infection than after $\mathrm{XC}$ infection at $45 \mathrm{dpi}$. The WAK2 (BraA06001041), WAK4 (BraA06001042), and WAK-like 9 (BraA08003575) expressions were higher upon SF infection than upon $\mathrm{XC}$ infection at 4 and 45 dpi.

Incompatible strubbelig receptor family $3 \mathrm{SRF} 3$ alleles are required for an enhanced early immune response to pathogens (Alcázar et al. 2014). Here, strubbelig (BraA09000500, BraA06002540, and BraA09000534), SRF8 (BraA08001525) and SRF6 (BraA06001833) were up-regulated at $16 \mathrm{dpi}$ after SF infection but downregulated after $\mathrm{XC}$ infection at $45 \mathrm{dpi}$.

The nuclear shuttle protein-interacting kinase (NIK)
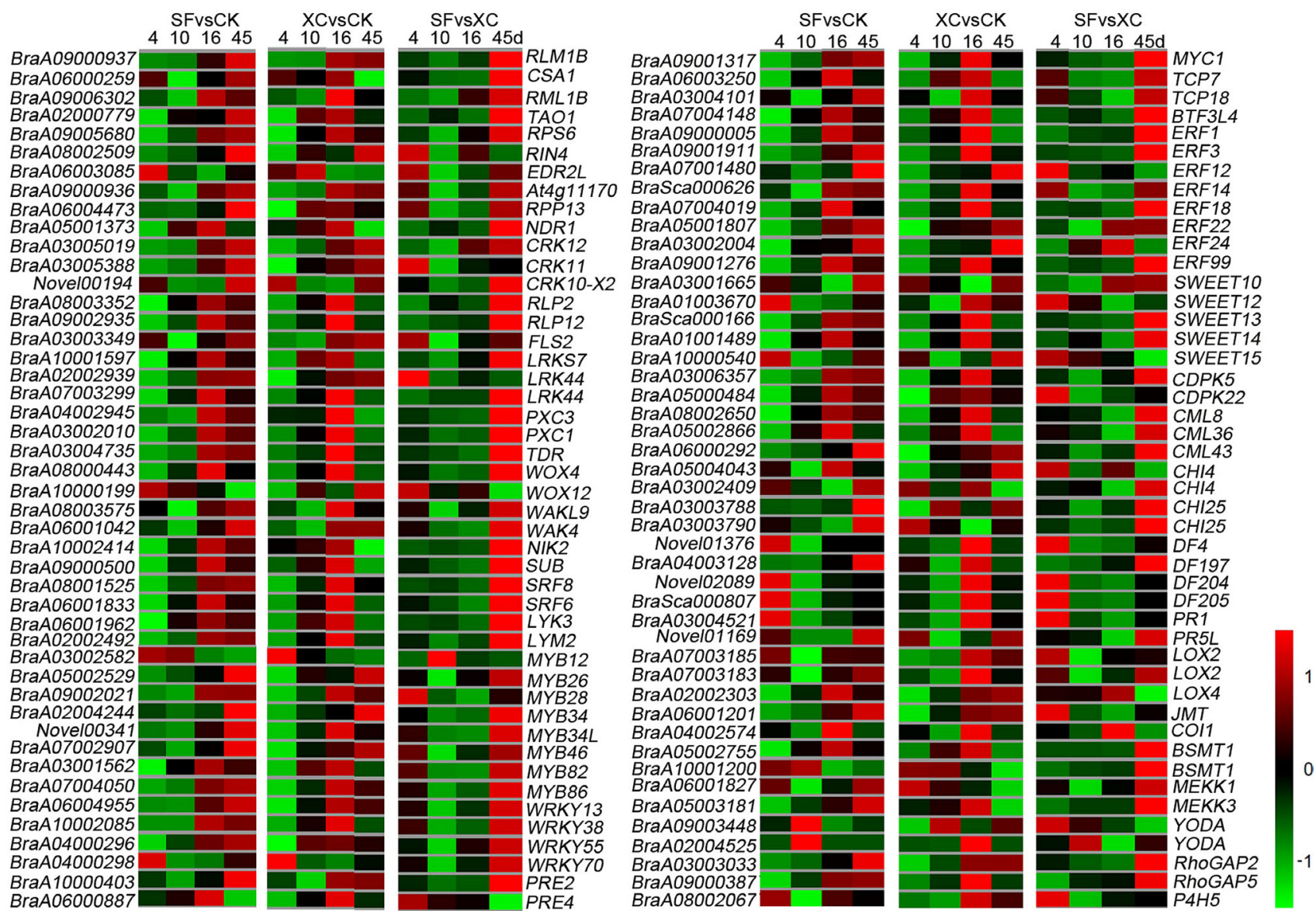

Fig. 4. Heat maps of differentially expressed genes (DEGs) for putative candidate genes assigned to clubroot-resistant genes in Brassica rapa determined at 4, 40,16, and 45 days after inoculation. Green indicates down-regulated DEGs, and red indicates up-regulated DEGs when compared Shifang (SF) with control (CK), Xichang (XC) with CK, and SF with XC. 
is a leucine-rich repeats-containing receptor-like serine/ threonine kinase and involved in plant development and defense (Santos et al. 2010). The NIK1 (BraA10000339), NIK2 (BraA06004385 and BraA10002414), and NIK3 (BraA01002380) were significantly induced already at 16 dpi and still up-regulated at 45 dpi upon SF infection. We suggest that these protein kinases might have an important role against the SF isolate infection.

Transcription factors related defense: In plants, transcriptional regulation is an important way for modulating defense responses. Genes with expressions more than 2-fold higher by SF vs. XC infection were selected including 19 myeloblastosis (MYB), 22 WRKY, 2 pathogen-responsive elements, 1 myelocytomatosis 1, 2 teosinte branched1/cycloidea/pcf (TCP), 1 basic transcription factor 3.

The WRKY transcription factors have been reported to regulate defense positively or negatively (Timmermann et al. 2017). We detected $22 W R K Y$ transcription factors, i.e., WRKY13, WRKY38, WRKY42, WRKY46, WRKY54, and $W R K Y 55$, whose expressions were 2-fold higher after $\mathrm{SF}$ infection than after $\mathrm{XC}$ infection. It is still unclear whether WRKYs act positively or negatively in response to the presence of $P$. brassicae, and thus the role of WRKY in clubroot resistance needs further study.

One function of TCP proteins is to regulate defense gene expression (Kim et al. 2014). We found that TCP7, 15, and 18 expressions were 2-fold higher upon SF $v s$. XC infection at $45 \mathrm{dpi}$. Therefore, these transcription factors with high expressions upon SF infection might play an important role against $P$. brassicae.

Cell wall modification: The cell wall biogenesis, especially the secondary cell wall formation and deposition, usually confers broad-spectrum resistance to pathogens. For example, Arabidopsis the walls are thin 1 confers broadspectrum resistance against vascular pathogens (Denancé et al. 2013). Over-expression of GhLacl, which functions as a lignin polymerization enzyme in Gossypium hirsutum can confer an enhanced resistance against pathogens $(\mathrm{Hu}$ et al. 2013).

Our analysis showed that 97 cell wall formation and deposition related genes up-regulated after the infections. The xyloglucan endotransglucosylase/hydrolase 5, $7,8,9,16,17,22,25,31,32$, and 33, fasciclin like arabinogalactan proteins $1,8,10,11$, and 17 , cellulose synthase A, B4 and D5, COBRA-like protein 6 , pectin methylesterases 1, 53, and 68, and pectin methylesterase inhibitors 3, 6, 16, 18, 44, 51, 61, and 64, subtilisin-like protease 1.2, 1.6, 1.7, 2.5, 3.3, 3.5, walls are thin 1 related protein, galacturonosyltransferase 1, cinnamyl alcohol dehydrogenase 6 and 7, laccase 4, 10,11,12, and 22, and irregular xylem 9, 10, 14H, and 15. The 3-ketoacyl-CoA synthase 6, required for synthesis of epicuticular waxes, at $4 \mathrm{dpi}$, trichome birefringence like 6, 18, 33, 36, 37, and 39 at 16 and $45 \mathrm{dpi}$, callose synthase 3 and 9, and plasmodesmata callose-binding protein 1, 3, 4, and 5 at 45 dpi had higher expressions upon SF infection than $\mathrm{XC}$ infection.

In this analysis, cell wall formation and deposition related genes were mainly up-regulated at 16 and $45 \mathrm{dpi}$, but not at the early infection stages, indicating the cell wall modification enhanced the resistance to SF isolate during the late infection periods.

Reactive oxygen species scavenging related genes exhibited higher expressions after SF infection compared

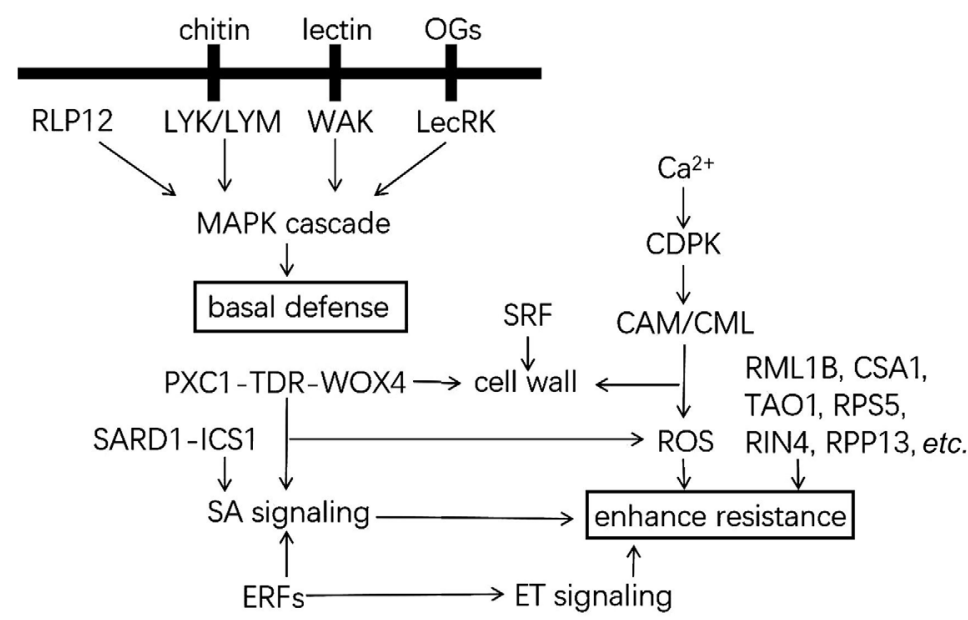

Fig. 5. A hypothetic working model for activation of the defense of the Plasmodiophora brassicae Shifang isolate in Brassica rapa. CAM/CML - calmodulin/calmodulin-like, CDPK - calcium-dependent protein kinase, CSA1 - constitutive shade-avoidance 1, ET - ethylene, ERFs - ethylene responsive factors, ICS1 - isochorismate synthase 1, LecRK - lectin receptor kinase, LYK/LYM - lysin motif receptor kinase / lysin motif, MAPK - mitogen-activated protein kinase, OGs - Oligogalacturonides, PXC1 - phloem intercalated with xylem-correlated 1, RIN4 - resistance to Pseudomonas syringae pv. maculicola interacting protein 4, RLP12 - receptor-like protein, RML1B - resistance to Leptosphaeria maculans 1B, ROS - reactive oxygen species, RPP13 - recognition of Peronospora parasitica 13 , RPS5 - ribosomal protein of the small subunit 5, SARD1 - systemic acquired resistance deficient 1, SA - salicylic acid, SRF - strubbelig receptor family, TAO1 - target of AvrB operation 1, TDR - tracheary element differentiation inhibitory factor receptor, WAK - wallassociated kinase; WOX4 - wuschel homeobox related 4. 
with XC infection. Glutaredoxin, L-ascorbate oxidase, peroxidase, catalase, and respiratory burst oxidase homolog protein $E$ were induced at 4 and 45 dpi.

Sugar transporters: Sugars will eventually be exported transporter (SWEET) proteins, a novel family of sugar transporters, act as important players in sucrose phloem loading. Due to the disruption of sugar efflux and changes in sugar distribution, SWEET can enhance the resistance to biotic and abiotic stresses (Li et al. 2017). In our data, we identified 12 SWEET genes, including 3 genes (BraA10000540, BraA01003670, and BraA06000647) at 4 dpi, 3 genes (BraA02000503, BraA06004193, and BraA09004936) at $10 \mathrm{dpi}, 5$ genes (BraA08002029, BraA01003908, BraSca000166, BraA06002331, and BraA01001489) at $45 \mathrm{dpi}$, and 1 gene (BraA03001665) at 16 and 45 dpi, whose expressions were 2-fold higher upon $\mathrm{SF}$ infection than $\mathrm{XC}$ infection. The disruption of sugar efflux and changes in sugar distribution in plant roots might influence P. brassicae infection and extension.

Calcium and calcium-dependent protein kinases: Calcium is an essential second messenger and calciumdependent protein kinases (CDPKs) and CDPK-related kinases (CRKs) regulate plant responses to abiotic and biotic stresses (Wang et al. 2016). Here we found that the expressions of three mitochondrial calcium uniporter, three calmodulin-like, and three calcium binding genes were more than 2-fold higher at $45 \mathrm{dpi}$ when comparing $\mathrm{SF}$ infection with $\mathrm{XC}$ infection. The CDPK15 was upregulated at $4 \mathrm{dpi}$. The CDPK-related kinase 4 and 7 were induced upon SF infection at $16 \mathrm{dpi}$; CDPK5 and CDPK22 at 16 and 45 dpi.

Chitinase response to $\boldsymbol{P}$. brassicae infection: Most chitinases can degrade chitin, which is the main component of cell walls in P. brassicae (Schwelm et al. 2015). Six endochitinases were identified. The BraA05004043 was highly expressed over the time course of SF infection. The BraA03003790 and BraA03002409 were up-regulated after $\mathrm{SF}$ infection at 4 and $45 \mathrm{dpi}$; the expression was about 4 to 7 -fold higher after SF vs. XC infection at $45 \mathrm{dpi}$.

Pathogenesis-related (PR) proteins and defensin-like proteins: One of the features of plant defense responses is the production of PR proteins (Van Loon et al. 1999). Pathogenesis-related proteins $P R 1$ and $P R 5$, and defensinlike proteins 4, 37, 196, 197, 204, and 205 were induced. The expressions of PR1 (BraA03004521) was 11-fold higher, PR5 (BraA07004010) was 4-fold higher at $4 \mathrm{dpi}$, PR1 (BraA03006209, BraA03006206, and BraA08001998) and PR5 (Novel01169) were 2 to 3 -fold higher at $45 \mathrm{dpi}$ after SF vs. XC infection. These PR proteins are most likely involved in $B$. rapa resistance to $P$. brassicae.

Salicylic acid (SA), jasmonic acid (JA) and ethylene (ET) signaling pathways: $P$. brassicae is an obligate biotrophic protist. Plant resistance to biotrophic pathogens is believed to be controlled by the SA-mediated signaling pathway. In this study, non-inducible immunity 1 , phytoalexin deficient 4, isochorismate synthase 1, and systemic acquired resistance deficient 1 were induced. The ethylene responsive factors (ERFs) can regulate the SA and JA/ET signaling pathways, and they are responsible for generating tolerance to biotic stresses in plants. We identified $41 E R F$ s including ERF 1, 3, 12, 13, 14, 16, 18, $19,20,21,22,24,25,34,35,38,43,54,95,107,115$, and 122, aintegumenta, aintegumenta-like, cytokinin response factor, related to apetala 2, and wax inducer 1 . Thus, numerous ERFs were involved in plant responses to $P$. brassicae during the late infection stages and could be considered as potential candidate genes for further functional validations.

Secondary metabolites associated with plant defense: Secondary metabolites are involved in diverse stressinduced responses. Previous studies have reported that nodulin proteins are associated with plant defense in Medicago truncatula (Gamas et al. 1998). Serine carboxypeptidase-like protein is required for disease resistance in oat (Mugford et al. 2009). Trehalose-6phosphate synthase/phosphatase is involved in resistance to Botrytis cinerea and Pseudomonas syringae pv. tomato DC3000 in tomato (Zhang et al. 2016). Thaumatin-like protein, germin-like protein and patatin-like protein 2 (PLP2) can enhance resistance to some pathogens (La Camera et al. 2009, Liu et al. 2016). Here, we found that eight early nodulin-like proteins, four serine carboxypeptidase, five thaumatin-like proteins, two patatin-like proteins with higher expressions at 16 dpi after both SF and XC infections, and still maintained higher at 45 dpi after SF infection. The secondary metabolism might play roles for resistance against SF infection extension.

\section{Conclusions}

In this study, we identified a number of genes that might contribute to defense against $P$. brassicae through transcriptome analysis on a time course of infection. Most of the genes up-regulated in cortical stage of infection by $\mathrm{SF}$ and $\mathrm{XC}$ isolates were down-regulated at $4 \mathrm{dpi}$, which indicated that the defense response activated during the cortical infection period were suppressed during the root hair infection period. This may explain that the root hair infection can occur in various plants including resistant, susceptible, or non-hosts plants, and the cortical infection only occurs in the susceptible plants. Based on the continuous defense against $P$. brassicae, such as the restriction of $P$. brassicae proliferation, extension, and secondary tissue development during the cortical infection period, the symptom finally developed into two different types.

Here, we compared the data from SGGs sample of SF infection with spindle galls sample of XC infection, and proposed a working hypothesis for the defense against SF infection (Fig. 5). Firstly, there are a few receptors, such as CDPK, lysin motif receptor kinase / lysin motif, WAK, and LecRK that recognize $\mathrm{Ca}^{2+}$, chitin, oligogalacturonides, and lectin. Other pattern recognition receptors $R L P 12$, and 
flagelin-sensitive 2 are highly induced upon SF infection, so a series of defense pathways may be activated. Subsequently, genes involved in MAPK cascades are also up-regulated, which in turn presumably leads to activation of the basal defense. Secondly, there is a effector-triggered immunity response in $B$. rapa $\mathrm{T} 1-145$ to $\mathrm{SF}$ infection, which is mediated by NB-LRR disease resistance proteins resistance to leptosphaeria maculans $1 \mathrm{~B}$, constitutive shade-avoidance 1, TAO1, RPS6, RIN4, enhanced disease resistance $2 L$, and recognition of Peronospora parasitica 13 like protein 4 . In addition, a batch of genes including $S R F, M Y B$, and $P X C 1-T D R-W O X$ are up-regulated, which probably lead to the secondary cell wall formation. Many $E R F s, W R K Y$, and $M Y B$ transcript factors are induced, most of which can act as transcriptional activators or repressors of SA, JA, and ET signaling, reactive oxygen species, cell wall integrity, etc. According to this model, these genes could be the candidates for engineering disease resistance by transgenic approaches.

\section{References}

Alcázar, R., Von Reth, M., Bautor, J., Chae, E., Weigel, D., Koornneef, M., Parker, J.E.: Analysis of a plant complex resistance gene locus underlying immune-related hybrid incompatibility and its occurrence in nature. - PLoS Genet. 10: e1004848, 2014

Audic, S., Claverie, J.M.: The significance of digital gene expression profiles. - Genome Res. 7: 986-995, 1997.

Ashburner, M., Ball, C.A., Blake, J.A., Botstein, D., Butler, H., Cherry, J.M.: Geneontology: tool for the unification of biology. - Nat. Genet. 25: 25-29, 2000

Chen, J., Pang, W., Chen, B., Zhang, C., Piao, Z.: Transcriptome analysis of Brassica rapa near-isogenic lines carrying clubroot-resistant and -susceptible alleles in response to Plasmodiophora brassicae during early infection. - Front Plant Sci. 6: 1183, 2016.

Ciaghi, S., Schwelm, A., Neuhauser, S.: Transcriptomic response in symptomless roots of clubroot infected kohlrabi (Brassica oleracea var. gongylodes) mirrors resistant plants. - BMC Plant Biol. 19: 288, 2019.

Crisp, P., Crute, I.R., Sutherland, R.A., Angell, S.M., Bloo, R.K.: The exploitation of genetic resources of Brassica oleracea in breeding for resistance to clubroot Plasmodiophora brassicae. - Euphytica 42: 215-226, 1989.

Delteil, A., Gobbato, E., Cayrol, B., Estevan, J., Michel-Romiti, C., Dievart, A.: Several wall- associated kinases participate positively and negatively in basal defense against rice blast fungus. - BMC Plant Biol. 16: 17, 2016.

Denancé, N., Ranocha, P., Oria, N., Barlet, X., Rivière, M.P., Yadeta, K.A.: Arabidopsis wat1 (walls are thin1)-mediated resistance to the bacterial vascular pathogen, Ralstonia solanacearum, is accompanied by cross-regulation of salicylic acid and tryptophan metabolism. - Plant J. 73: 225-239, 2013.

Devos, S., Laukens, K., Deckers, P., Van Der Straeten, D., Beeckman, T., Inzé, D., Van Onckelen, H., Witters, E., Prinsen, E.: A hormone and proteome approach to picturing the initial metabolic events during Plasmodiophora brassicae infection on Arabidopsis. -Mol. Plant Microbe Interact. 19: 1431-1443, 2006.

Eitas, T.K., Nimchuk, Z.L., Dangl, J.L.: Arabidopsis TAO1 is a TIR-NB-LRR protein that contributes to disease resistance induced by the Pseudomonas syringae effector AvrB. - Proc. nat. Acad. Sci. USA 105: 6475-6480, 2008

Gamas, P., De Billy, F., Truchet, G.: Symbiosis-specific expression of two Medicago truncatula nodulin genes, MtN1 and $M t N 13$, encoding products homologous to plant defense proteins. - Mol. Plant Microbe Interact. 11: 393-403, 1998.

Holtz, M.D., Hwang, S.F., Strelkov, S.E.: Genotyping of Plasmodiophora brassicae reveals the presence of disinct populations. - BMC Genomics 19: 254, 2018.

Hu, Q., Min, L., Yang, X., Jin, S., Zhang, L., Li, Y.: Laccase GhLac1 modulates broad-spectrum biotic stress tolerance via DAMP-triggered immunity. - Plant Physiol. 176: 1808-1823, 2017.

Ji, R., Zhao, L., Xing, M., Shen, X., Bi, Q., Peng, S., Feng, H.: Infection of Plasmodiophora brassicae in Chinese cabbage. Genet. mol. Res. 13: 10976-10982, 2014.

Jubault, M., Lariagon, C., Taconnat, L., Renou, J.P., Gravot, A., Delourme, R., Manzanares- Dauleux, M.J.: Partial resistance to clubroot in Arabidopsis is based on changes in the host primary metabolism and targeted cell division and expansion capacity. - Funct. integr. Genomics 13: 191-205, 2013.

Kageyama, K., Asano, T.: Life cycle of Plasmodiophora brassicae. - J. Plant Growth Regul. 28: 203-211, 2009.

Kim, S.H., Son, G.H., Bhattacharjee, S., Kim, H.J., Nam, J.C., Nguyen, P.D.: The Arabidopsis immune adaptor SRFR1 interacts with TCP transcription factors that redundantly contribute to effector-triggered immunity. - Plant J. 78: 978789, 2014

La Camera, S., Balague, C., Gobel, C., Geoffroy, P., Legrand, M., Feussner, I., Roby, D., Heitz, T.: The Arabidopsis patatinlike protein 2 (PLP2) plays an essential role in cell death execution and differentially affects biosynthesis of oxylipins and resistance to pathogens. - Mol Plant Microbe Interact. 22: 469-481, 2009.

Li. K., Hegarty, J., Zhang, C., Wan, A., Wu, J., Guedira, G.B.: Fine mapping of barley locus Rps6 conferring resistance to wheat stripe rust. - Theor. appl. Genet. 129: 845-859, 2016.

Li, Y., Wang, Y., Zhang, H., Zhang, Q., Zhai, H., Liu, Q., He, S.: The plasma membrane-localized sucrose transporter IbSWEET10 contributes to the resistance of sweet potato to Fusarium oxysporum. - Front Plant Sci. 8: 197, 2017.

Li, H., Li, X., Xuan, Y., Jiang, J., Wei, Y., Piao, Z.: Genome wide identification and expression profiling of SWEET genes family reveals its role during Plasmodiophora brassicae induced formation of clubroot in Brassica rapa. - Front Plant Sci. 9: 207, 2018.

Liu, Q., Yang, J., Yan, S., Zhang, S., Zhao, J., Wang, W.: The germin-like protein OsGLP2-1 enhances resistance to fungal blast and bacterial blight in rice. - Plant mol. Biol. 92: 411423, 2016.

Ludwig-Müller, J.: Auxin homeostasis, signaling, and interaction with other growth hormones during the clubroot disease of Brassicaceae. - Plant Signal Behav. 9: e28593, 2014.

Luo, X., Xu, N., Huang, J., Gao, F., Zou, H., Boudsocq, M.: A lectin receptor- like kinase mediates pattern-triggered salicylic acid signaling. - Plant Physiol. 174: 2501-2514, 2017.

Mugford, S.T., Qi, X., Bakht, S., Hill, L., Wegel, E., Hughes, RK.: A serine carboxypeptidase- like acyltransferase is required for synthesis of antimicrobial compounds and disease resistance in oats. - Plant Cell 21: 2473-2484, 2009.

Mortazavi, A., Williams, B.A., McCue, K., Schaeffer, L., Wold, B.: Mapping and quantifying mammalian transcriptomes by RNA-Seq. - Nat. Methods 5: 621-628, 2008.

Osaki, K., Tanaka, S., Ito, S.: Pathogenicity of Plasmodiophora brassicae populations from small, spheroid, resistant-type clubroot galls on roots of clubroot-resistant cultivars of Chinese cabbage (Brassica rapa L. subsp. pekinensis). - J. 
gen. Plant Pathol. 74: 242-245, 2008.

Peng, Y., Huang, Y., Liu, Z.L., Yang, H., Shang, J.: Occurrence and morphology of small spheroid galls on clubroot-resistant Chinese cabbage. - Eur. J. Plant Pathol. 145: 591-599, 2016.

Piao, Z.Y., Ramchiary, N., Lim, Y.P.: Genetics of clubroot resistance in Brassica species. - J. Plant Growth Regul. 28: 252-264, 2009.

Pitino, M., Armstrong, C.M., Duan, Y.: Rapid screening for citrus canker resistance employing pathogen-associated molecular pattern-triggered immunity responses. - Hort. Res. 2: 15042, 2015.

Rennie, D.C., Manolii, V.P., Plishka, M., Strelkov, S.E.: Histological analysis of spindle and spheroid root galls caused by Plasmodiophora brassicae. - Eur. J. Plant Pathol. 135: 771-781, 2013.

Santos, A. A., Lopes, K.V.G., Apfata, J.A.C., Fontes, E.P.: NSP-interacting kinase, NIK: a transducer of plant defence signalling. - J. exp. Bot. 14: 3839-3845, 2010.

Schwelm, A., Fogelqvist, J., Knaust, A., Jülke, S., Lilja, T., Bonilla-Rosso, G.: The Plasmodiophora brassicae genome reveals insights in its life cycle and ancestry of chitin synthases. - Sci. Rep. 5: 11153, 2015.

Siemens, J., Keller, I., Sarx, J., Kunz, S., Schuller, A., Nagel, W., Schmülling, T., Parniske, M., Ludwig-Müller, J.: Transcriptome analysis of Arabidopsis clubroots indicate a key role for cytokinins in disease development. - Mol. Plant Microbe Interact. 19: 480-494, 2006.

Sun, X., Greenwood, D.R., Templeton, M.D., Libich, D.S., McGhie, T.K., Xue, B.: The intrinsically disordered structural platform of the plant defence hub protein RPM1-interacting protein 4 provides insights into its mode of action in the host-pathogen interface and evolution of the nitrate-induced domain protein family. - FEBS J. 281: 3955-3979, 2014.

Suwabe, K., Tsukazaki, H., Iketani, H., Hatakeyama, K., Kondo, M.: Simple sequence repeat-based comparative genomics between Brassica rapa and Arabidopsis thaliana: the genetic origin of clubroot resistance. - Genetics 173: 309-319, 2006.

Tanaka, S., Ito, S.: Pathogenic and genetic diversity in Plasmodiophora brassicae (clubroot) from Japan. - J. gen. Plant Pathol. 79: 297-306, 2013.

Timmermann, T., Armijo, G., Donoso, R., Seguel, A., Holuigue, L., González, B.: Paraburkholderia phytofirmans PsJN protects Arabidopsis thaliana against a virulent strain of Pseudomonas syringae through the activation of induced resistance. - Mol. Plant Microbe Interact. 30: 215-230, 2017.
Trapnell, C., Pachter, L., Salzberg, S.L.: TopHat: discovering splice junctions with RNA-Seq. - Bioinformatics 25: 11051111, 2009.

Van Loon, L.C., Van Strien, E.A.: The families of pathogenesisrelated proteins: their activities, and comparative analysis of PR-1 type proteins. - Physiol. mol. Plant Pathol. 55: 85-97, 1999.

Wang, J., Kucukoglu, M., Zhang, L., Chen, P., Decker, D., Nilsson, O.: The Arabidopsis LRR-RLK, PXC1, is a regulator of secondary wall formation correlated with the TDIF-PXY/ TDR-WOX4 signaling pathway. - BMC Plant Biol. 13: 94, 2013.

Wang, J.P., Xu, Y.P., Munyampundu, J.P., Liu, T.Y., Cai, X.Z.: Calcium-dependent protein kinase $(C D P K)$ and CDPKrelated kinase $(C R K)$ gene families in tomato: genome-wide identification and functional analyses in disease resistance. Mol. Genet. Genomics 291: 661-676, 2016.

Wang, L., Feng, Z., Wang, X.: DEGseq: an R package for identifying differentially expressed genes from RNA-seq data[J]. - Bioinformatics 26: 136-138, 2010.

Williams, P.H.: A system for the determination of races of Plasmodiophora brassicae that infect cabbage and rutabaga. Phytopathology 56: 624-626, 1996.

Wu, J., Mao, X., Cai, T., Luo, J., Wei, L.: KOBAS server: a web-based platform for automated annotation and pathway identification. - Nucl. Acids Res. 34: 720-724, 2006.

Xue, S., Cao, T., Howard, R.J., Hwang, S.F., Strelkov, S.E.: Isolation and variation in virulence of single-spore isolates of Plasmodiophora brassicae from Canada. - Plant Dis. 92: 456-462, 2008.

Young, M.D., Wakefield, M.J., Smyth, G.K., Oshlack, A.: Gene ontology analysis for RNA-seq: accounting for selection bias. - Genome Biol. 11: 1-12, 2010.

Zhang, H., Hong, Y., Huang, L., Liu, S., Tian, L., Dai, Y., Cao, Z., Huang, L., Li, D., Song, F.: Virus-induced gene silencingbased functional analyses revealed the involvement of several putative trehalose-6- phosphate synthase /phosphatase genes in disease resistance against Botrytis cinerea and Pseudomonas syringae pv. tomato DC3000 in tomato. - Front Plant Sci. 7: 1176, 2016.

Zhao, Y., Bi, K., Gao, Z., Chen, T., Liu, H., Xie, J., Cheng, J., Fu, Y., Jiang, D.: Transcriptome analysis of Arabidopsis thaliana in response to Plasmodiophora brassicae during early infection. - Front. Microbiol. 8: 673, 2017. 